\author{
Nathalie Favre \\ Jean-Nicolas Despland \\ Yves de Roten \\ Martin Drapeau \\ Mathieu Bernard \\ Friedrich Stiefel
}

\section{Psychodynamic aspects of communication skills training: a pilot study}

Received: 3 May 2006

Accepted: 16 August 2006

Published online: 17 October 2006

(C) Springer-Verlag 2006

N. Favre · F. Stiefel $(\bowtie)$

Psychiatry Service,

University Hospital (CHUV),

Lausanne, Switzerland

e-mail: Frederic.Stiefel@chuv.ch

Tel.: +41-21-3141090

Fax: +41-21-3141086

J.-N. Despland · Y. de Roten ·

M. Bernard

Division of Psychotherapy Research,

Department of Psychiatry,

Lausanne, Switzerland

M. Drapeau

The Institute of Community

and Family Psychiatry,

Sir Mortimer B. Davis Jewish General

Hospital and McGill University,

Montreal, Canada

\begin{abstract}
Goals of work: Communication between patients and oncology clinicians is a key element of cancer care. Emotionally charged consultations may trigger clinicians' defense mechanisms, protecting them from painful emotions. Defense mechanisms, however, may also hamper the recognition of patients' suffering. This pilot study aims to explore clinicians' defense mechanisms observed in communication skills training (CST). Patients and methods: A verbatim transcription of videotaped interviews with simulated patients were evaluated before $(N=10)$ and after CST $(N=10)$ with the defense mechanism rating scales (DMRS). Main results: A wide variety of defense mechanisms were observed such as obsessional (e.g.
\end{abstract}

intellectualisation) or disavowal (e.g. denial or projection). Immature defense mechanisms decreased after CST. Conclusions: A wide variety of defense mechanisms are operant in oncology clinicians facing challenging interviews with simulated patients. Defense mechanisms may be modified by CST.

Keywords Communication skills training $\cdot$ Defense mechanisms · Cancer - DMRS $\cdot$ Simulated patients

\section{Introduction}

Giving bad news in cancer care may provoke painful feelings, such as of powerlessness, fear of being blamed or grief $[3,9,11,12,20]$, that may, in turn, trigger defense mechanisms in clinicians. Defense mechanisms can be defined as psychological mechanisms that mediate between an individual's needs and external reality [27]. They help to cope with emotions evoked by internal and external stressors, function without conscious effort and often follow lawful patterns. Defenses can be categorized ranging from immature defenses, such as denial or projection, to more mature defenses such as sublimation or intellectualisation [21, 23]. Defense mechanisms can be studied empirically, and an important body of research focusing on this topic now exists in the field of psychotherapy [4].

While defenses protect clinicians from painful emotions, if not adapted, they can also seriously hamper communication, empathy and recognition of a patient's psycho- logical needs [31]. For example, clinicians' defense mechanisms may induce abrupt transitions during the interview, an inadequate focus on physical symptoms, an increase of closed questions, early comforting, blank denial of patients' distress, aggressiveness or detachment [14]. Such elements, on the other hand, are often mentioned as affecting the patient's psychological adjustment $[13,17$, 28], quality of life, adherence to treatment, pain control [28], information recall [13] and satisfaction with care [19]. Moreover, they can also affect the well-being of clinicians themselves: oncology clinicians report stress, lack of job satisfaction and emotional burnout related to their feelings of communicating inadequately [7].

Only a minority of oncologists have received formal training in communication, and the training provided is commonly inadequate [10]. Communication in oncology calls for specific skills and a training that is tailored to the daily clinical practice. Specific communication skills training (CST) for oncology clinicians has therefore been developed over the last decade [16]. Closely related to 
clinical practice, it provides an opportunity to experience communication and to interact with trainers and peers by means of case-history discussions, analyses of audioor videotaped interviews with simulated patients and structured role play. These techniques allow to heighten self-awareness, to identify communicational difficulties and to practise new ways of communication [10].

There is increasing evidence that training is efficient in improving objective communication performance $[2,8,18$, $22]$. According to these studies, participants use more focused and open questions after training; they express more empathy and give more appropriate responses to the patient's cues [10]. While evidence exists for the effectiveness of CST, it remains unknown how CST modifies communication and leads to change. Our group, which trained over 100 oncology clinicians over the last 5 years [14], has observed that CST participants seem to become less anxious when facing challenging situations and therefore more adequate in relating to patients. The modification of clinicians' defense mechanisms may be the key for change.

Based on the existing body of research in psychotherapy, we hypothesize that defense mechanisms can be identified in oncology clinicians with satisfactory reliability. Secondly, we hypothesize that, with an increased feeling of competence with regard to communication, clinicians use more adaptive defense mechanisms, therefore reducing negative consequences on communication. In order to test a possible approach to examine this hypothesis, we conducted a pilot study, which is presented in this article.

\section{Patients and methods}

\section{Setting of the study/CST}

In Switzerland, a national task force has developed a structured, manual-based CST for oncology clinicians; conducted since 1999 in the German-, French- and Italianspeaking part of the country [16], it has meanwhile become mandatory for the specialisation in oncology. This CST is based on three core elements: (1) case-history discussion, (2) analyses of videotaped interviews with simulated patients and (3) structured role play, and consists of a 2-day retreat for up to ten physicians and oncology nurses and is conducted by two trainers also responsible for the follow-up. Follow-up consists of four to six individual supervisions during which the participants can discuss cases that have challenged them from a communicational point of view; some participants also choose to analyse with the trainers their video- or audiotaped medical consultations. After 6 months, another half-day training session and a second videotaped interview with a simulated patient take place. A random sample of these videotaped interviews at the beginning of the CST and 6 months later was utilised in this study.

\section{Sample}

Ten oncology physicians and nurses participated in CST between 1999 and 2003. The sample consisted of nine women and one man (seven nurses and three physicians) with a mean age of $39.3(\mathrm{SD}=10.7)$. An informed consent concerning the utilisation of the videotaped interview was obtained.

\section{Measurements}

The defense mechanism rating scale (DMRS) [24] is an observer-rated method for the rating of defense mechanisms, which is utilised for audio or video recording or the written transcription of various forms of interviews or therapy sessions. Many studies have supported the validity and reliability of the method [15, 25, 26, 29].

The instrument includes a total of 30 defense mechanisms assigned to seven hierarchical levels of defensive functioning (see Table 1): mature, obsessional, other neurotic, narcissistic (minor image distorting), disavowal, borderline (major image distorting) and action defenses. Each level includes three to eight individual defenses, which can be weighted according to its level of maturity and summed up to an overall defensive functioning score (ODF). Defense mechanisms can also be categorized into three broader levels: mature, neurotic and immature, according to Vaillant's classification [32].

Table 1 DMRS hierarchy of adaptation

\begin{tabular}{lll}
\hline Levels (3) & Levels (7) & Defense mechanisms (30) \\
\hline Mature & Mature & Affiliation, altruism, anticipation, humor, self-assertion, self-observation, sublimation, suppression \\
Neurotic & Obsessional & Intellectualisation, isolation, undoing \\
& Other neurotic & Repression, dissociation, reaction formation, displacement \\
Immature & Narcissistic & Devaluation of self, devaluation of others, idealization of self, idealization of others, omnipotence \\
& Disavowal & Denial, projection, rationalization, autistic fantasy \\
& Borderline & Splitting of other's images, splitting of self-images, projective identification \\
& Action & Acting out, hypochondriasis, passive-aggression \\
\hline
\end{tabular}


It has been necessary to adapt DMRS to rate therapists' defenses. An addendum to the manual was developed to clarify possible difficulties in ratings (DMRS-Therapist). The psychometric characteristics of the DMRS-T are equivalent to the DMRS for the patients [5].

\section{Main results}

The applicability and face validity of the DMRS-T for the rating of clinician defenses have been confirmed in this pilot study. The inter-rater reliability (ICC) for defense categories was $r=0.82$.

The number of defense mechanisms observed in the interviews ranged from 10 to $35(m=23.4, \mathrm{SD}=6.35)$. Among the 30 defense mechanisms measured by the instrument, 23 were coded at least once, and all defensive levels were represented. In addition, inter- and intraindividual variability was observed. Defense mechanisms varied between clinicians and also between interviews for the same clinician. Most frequently observed defenses were obsessional (mainly intellectualisation and annulation), other neurotic (mainly reaction formation and displacement) and disavowal (rationalization, denial and projection).

The Wilcoxon rank test was used for analysis. Frequencies of defense levels and ODF are shown in Table 2. First of all, the ODF score increased significantly after CST $(p<0.05)$. A significant decrease of immature defenses was observed when using Vaillant's categorization $(p<0.05)$. Among the seven levels of the DMRS, the borderline defense level showed a significant decrease.

On the individual level, the ODF increased for eight out of ten clinicians (Fig. 1).

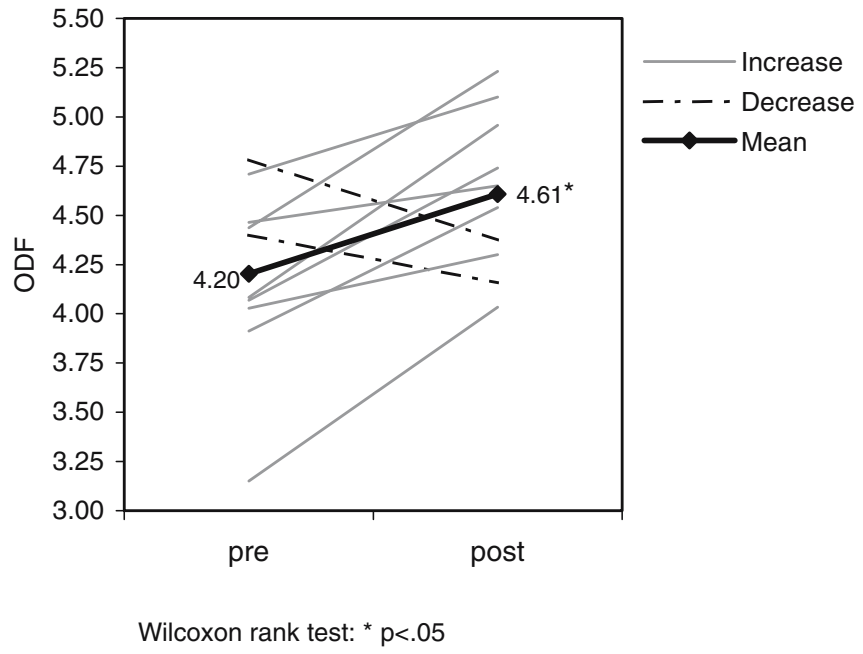

Fig. 1 Impact of CST $(N=10)$. Wilcoxon rank test: ${ }^{*} p<0.05$

\section{Discussion}

A variety of defense mechanisms were observed among clinicians interviewing patients in CST. This confirms the hypothesis that clinicians may protect themselves from painful emotions (anxiety, guilt, powerlessness) generated by the interaction with patients, by mobilizing defense mechanisms. Moreover, the results suggest that changes can be observed over the course of a CST. The ODF score increases significantly, indicating a progression after CST towards more mature defenses, and immature defense mechanisms decrease significantly. This raises the question how defensive functioning can change after such a short intervention; however, psychotherapy research has already demonstrated that defenses may change even during very brief interventions [6].

Interestingly, the two clinicians for which the ODF decreases after CST are the two youngest of the sample $(<30$ years old $)$.

Table 2 Frequency of defense levels and ODF before and after CST $(N=10)$

\begin{tabular}{lllllll}
\hline DMRS-T & & Levels & Pre & Post & $Z$ & $p$ value \\
\hline Defense levels & Mature & Mature & $2.4 \%$ & $6.5 \%$ & 1.48 & 0.139 \\
& Neurotic & Obsessional & $23.5 \%$ & $27.2 \%$ & 0.87 & 0.386 \\
& & Other neurotic & $27.1 \%$ & $30.0 \%$ & 0.51 & 0.959 \\
& & All neurotic & $50.6 \%$ & $57.2 \%$ & 1.33 & 0.185 \\
& Immature & Narcissistic & $9.0 \%$ & $6.7 \%$ & 0.87 & 0.386 \\
& & Disavowal & $25.3 \%$ & $22.7 \%$ & 0.97 & 0.333 \\
& & Borderline & $2.5 \%$ & $0.3 \%$ & 2.02 & $0.043 *$ \\
ODF & Action & $10.2 \%$ & $6.6 \%$ & 1.68 & 0.093 \\
\hline
\end{tabular}

$O D F$ overall defensive functioning, $Z$ Wilcoxon rank test ${ }^{*} p<0.05$ 
A more comprehensive study is under way to confirm these pilot results and to control for parameters of change such as repeated experience of role playing or growing clinical experience over time. This next study is designed as a controlled trial in which the evolution of defenses of participants in CST is compared to a control group, which performs two videotaped interviews with a simulated patient at a 6-month interval without any training [30]. Outcome measures are the (1) evolution of clinicians' defense mechanisms and (2) improvement in communication skills as measured by the Psychotherapy Process Q-Set (PQS) [1].

A confirmation of the hypothesis that improvement of communication skills is mediated by changes in clinicians' defense mechanisms would have the following implications. First, psychodynamic and relational elements in CST might be fostered. Second, a preparation of participants may have to be considered; up to now, anyone who was motivated to participate was included in our CST. On one occasion, a brief psychotic reaction of a participant occurred (unrealistic and severely distorted perception of a videotaped interview; the participant saw his deceased brother on the screen). This episode fortunately resolved within minutes and was without longer lasting consequences (Rousselle and Stiefel, 2003, personal communication). If in some participants major changes of defense mechanisms towards immature levels occur in CST, more attention to the defensive styles of participants will have to be introduced. Third, medical and nursing education might include the topic of defenses of health-care professionals in their curricula. Finally, the confirmation of our hypothesis would contribute to enhance the understanding of communication and interactions between clinicians and patients.

Acknowledgment This study has been supported by the Department of Psychiatry of the University Hospital, Lausanne, Switzerland and a grant by Oncosuisse (OCS-01595-08-2004).

\section{References}

1. Ablon JS, Jones EE (1998) How expert clinicians' prototypes of an ideal treatment correlate with outcome in psychodynamic and cognitive-behavioral therapy. Psychother Res 8:71-83

2. Baile WF, Kudelka AP, Beale EA, Glober GA, Myers EG, Greisinger AJ, Bast RC, Goldstein, MG, Novack D, Lenzi R (1999) Communication skills training in oncology: description and preliminary outcomes of workshops on breaking bad news and managing patient reactions to illness. Cancer 86:887-897

3. Baile WF, Beale EA (2001) Giving bad news to cancer patients: Matching process and content. J Clin Oncol 19:2575-2577

4. Despland JN, Drapeau M, de Roten Y (2001) Les mécanismes de défense: une pluralité de points de vue. Psychothérapies 3:113-121

5. Despland JN, Stiefel F, Favre N, Drapeau M, de Roten Y (2005) Adaptation of the defense mechanism rating scales for evaluating therapists' defense mechanisms: principles, reliability and face validity. Society for Psychotherapy Research, European and UK Chapters, Lausanne, 3 Mar 2005

6. Drapeau M, De Roten Y, Perry JC, Despland JN (2003) A study of stability and change in defense mechanisms during a brief psychodynamic investigation. J Nerv Ment Dis 191:496-502
7. Fallowfield L (1995) Can we improve the professional and personal fulfillment of doctors in cancer medicine? Br J Cancer 71:1132-1133

8. Fallowfield L, Lipkin M, Hall A (1998) Teaching senior oncologists communication skills: results from phase I of a comprehensive longitudinal program in the United Kingdom. J Clin Oncol 16:1961-1968

9. Fallowfield L, Jenkins V (1999) Effective communication skills are the key to good cancer care. Eur J Cancer 35:1592-1597

10. Fallowfield L, Jenkins V, Farewell V, Saul J, Duffy A, Eves R (2002) Efficacy of a cancer research UK communication skills training model for oncologists: a randomised controlled trial. The Lancet 359:650-656

11. Fallowfield L, Jenkins V (2004) Communicating sad, bad and difficult news in medicine. The Lancet 363:312-319

12. Florio GA, Donnelly JP, Zevon MA (1998) The structure of work-related stress and coping among oncology nurses in high-stress medical settings: a transactional analysis. J Occup Health Psychol 3:227-242

13. Ford S, Fallowfield L, Lewis S (1996) Doctor-patient interactions in oncology. Soc Sci Med 42:1511-1519

14. Guex P, Stiefel F, Rousselle I (2002) La communication: Un élément central en cancérologie. Revue Francophone de Psycho-Oncologie 1:43-46
15. Guldberg CA, Hoglend P, Perry JC (1993) Scientific methods for assessing psychological defences. Nordic Journal of Psychiatry 47:435-446 Taylor \& Francis, United Kingdom (peer reviewed journal)

16. Kiss A (1999) Communication skills training in oncology: a position paper. Ann Oncol 10:899-901

17. Lerman C, Daly M, Walsh WP, Resch N, Seay J, Barsevick A, Birenbaum L, Heggan T, Martin G (1993) Communication between patients with breast cancer and health care providers: determinants and implications. Cancer 72:2612-2620

18. Levinson W, Roter D (1993) The effects of two continuing medical education programs on communication skills of practicing primary care physicians. J Gen Intern Med 8:318-324

19. Loge JH, Kaasa S, Hytten K (1997) Disclosing the cancer diagnosis: the patients' experiences. Eur J Cancer 33:878-882

20. Meier DE, Back AL, Morrison RS (2001) The inner life of physicians and care of the seriously ill. JAMA 286:3007-3014

21. OPD Arbeitskreis (Hrsg) (1996) Operationalisierte Psychodynamische Diagnostik. Grundlagen und Manual. Verlag Hans Huber, Bern 
22. Parle M, Maguire P, Heaven C (1997) The development of a training model to improve health professionals' skills, self-efficacy and outcome expectancies when communicating with cancer patients. Soc Sci Med 44:231-240

23. Perry JC, Cooper S (1989) An empirical study of defense mechanisms. Arch Gen Psychiatry 46:444-452

24. Perry JC (1990) Defense mechanism rating scale, 5th edn. Cambridge University, Boston

25. Perry JC, Kardos ME, Pagano CJ (1993) The study of defenses in psychotherapy using the defense mechanism rating scales (DMRS). In: Ehlers W, Draguns JG (eds) The concept of defense mechanisms in contemporary psychology. Springer, Berlin Heidelberg New York, pp 122-132
26. Perry JC (2001) A pilot study of defenses in adults with personality disorders. J Nerv Ment Dis $1: 651-660$

27. Perry JC, Guelfi JD, Despland JN, Hanin B (2004) L'étude empirique des mécanismes de défense est-elle possible? In: Perry J.C., Guelfi JD, Hanin B, Despland JN (eds) Echelle d'évaluation des mécanismes de défense. Traduction et adaptation française du defense mechanism rating scales. Masson, Paris, pp 1-32

28. Razavi D, Delvaux N, Marchal S, De Cock M, Farvacques C, Schlachmuylder JL (2000) Testing health care professionals' communication skills: the usefulness of highly emotional standardized role-playing sessions with simulators. Psychooncology 9:293-302

29. Skodol AE, Perry JC (1993) Should an axis for defense mechanisms be included in DSM-IV? Compr Psychiatry 34:108-119
30. Stiefel F, Despland JN, Favre N, Guex P (2004) Effects of communication skills training on oncology clinicians' communication styles and defense mechanisms. Oncosuisse (OCS-0159508-2004)

31. Stiefel F, Favre N, Despland JN, de Roten Y (2006) Communication skills training in oncology: it works - a review of the literature. In: Stiefel F (ed) Communication in cancer care. Springer, Berlin Heidelberg New York (in press)

32. Vaillant GE, Bond M, Vaillant CO (1986) An empirically validated hierarchy of defense mechanisms. Arch Gen Psychiatry 43:786-794 